\title{
Inferior vena cava variation predicts fluid responsiveness during dysrhythmias: a rational rearrangement of chairs on The Titanic
}

\author{
Rory J. Spiegel, Michael T. McCurdy \\ Division of Pulmonary and Critical Care, University of Maryland School of Medicine, Baltimore, MD, USA \\ Correspondence to: Michael T. McCurdy. Division of Pulmonary and Critical Care, University of Maryland School of Medicine, Baltimore, MD, USA. \\ Email: drmccurdy@gmail.com. \\ Provenance: This is an invited Editorial commissioned by Section Editor Guo-Wei Tu, MD, PhD (Department of Critical Care Medicine, Zhongshan \\ Hospital, Fudan University, Shanghai, China). \\ Comment on: Bortolotti P, Colling D, Colas V, et al. Respiratory changes of the inferior vena cava diameter predict fluid responsiveness in \\ spontaneously breathing patients with cardiac arrhythmias. Ann Intensive Care 2018;8:79.
}

Submitted Sep 29, 2018. Accepted for publication Oct 11, 2018.

doi: $10.21037 /$ atm.2018.10.36

View this article at: http://dx.doi.org/10.21037/atm.2018.10.36

Fluid responsiveness, or identifying those patients whose position on the Frank-Starling curve will improve with fluid administration, has been a fundamental component of caring for critically ill patients even before critical care medicine became a specialty. However, how to optimally predict fluid responsiveness and what to do about it, if identified, has vexed clinicians for years. Since a relatively recent shift in conceptualizing patients' needs for fluid administration from static [e.g., central venous pressure (CVP)] to dynamic variables, many modalities have been explored to optimally accomplish this task. However, many existing dynamic methods to determine fluid responsiveness are plagued by technical difficulties performing the assessment (e.g., passive leg raise in an obese patient), costs associated with acquiring the necessary equipment [e.g., stroke volume (SV) variation-monitoring devices], insufficient validation in patient populations with certain pathology (e.g., dysrhythmias), or the need to administer unnecessary and potentially harmful therapies to obtain a diagnosis (e.g., intravenous fluid challenge). With its widespread adoption throughout intensive care units (ICUs), bedside ultrasound (US) is commonly used to predict fluid responsiveness by assessing the changing diameter of the inferior vena cava (IVC) with respiratory variation.

A recent article published by Bortolotti et al. in the Annals of Intensive Care elegantly examined the use of IVC ultrasound to predict fluid responsiveness in spontaneously breathing patients with irregular cardiac rhythms (1).
Patients in one of two French ICUs were included in the study if they were spontaneously breathing, presented with an irregular cardiac rhythm [defined as either atrial fibrillation or recurrent atrial extrasystoles (i.e., $>6 / \mathrm{min}$ )], and the treating physician decided a fluid bolus was clinically indicated.

The authors performed a thorough ultrasonographic assessment of patients prior to the fluid bolus, measuring velocity time integral (VTI) and left ventricular outflow tract diameter to determine $\mathrm{SV}$ and IVC collapsibility (cIVC), as defined by the difference between IVC diameter at end-expiration (eIVC) and end-inspiration (iIVC), throughout the respiratory cycle to determine the likelihood of a patient's preload responsiveness. Following volume expansion (VE) with a $500 \mathrm{~mL}$ bolus of $4 \%$ gelatin, the authors reassessed the VTI in order to calculate a change in SV. Patients were classified as fluid responsive if the SV increased by $\geq 10 \%$. The authors attempted to control for patients' varying inspiratory efforts by evaluating the change in IVC diameter between each patient's natural respiratory cycle and a deep standardized respiratory cycle. The deep standardized inspiration consisted of a brief and continuous inspiration to generate a minimum buccal pressure of -5 to $-10 \mathrm{mmH}_{2} \mathrm{O}$, followed by passive exhalation. Diaphragmatic excursion was evaluated using bedside ultrasound (US) during both spontaneous (IVC-sp) and standardized (IVC-st) breathing cycles to better characterize individual patient variation in respiratory effort. 
Of the 85 patients meeting inclusion criteria, 30 were excluded due to failure to capture the reference standard VTI or measure the IVC diameter. Fifty-five patients were included in the final analysis, $29(53 \%)$ had atrial fibrillation and $26(47 \%)$ had frequent extrasystoles. A total of $29(53 \%)$ patients were categorized as fluid responders. The cIVC was significantly greater in responders $v s$. nonresponders. Area under the ROC curves of cIVC and iIVC during spontaneous breathing were both 0.93 (95\% CI, 0.86-1.00). A change in cIVC $>39 \%$ prior to $\mathrm{VE}$ predicted fluid responsiveness with a sensitivity of 0.93 (95\% CI, 0.77-0.99) and a specificity of 0.88 (95\% CI, 0.69-0.97). Likewise, an iIVC-st $<11 \mathrm{~mm}$ prior to $\mathrm{VE}$ predicted fluid responsiveness with a sensitivity of 0.83 (95\% CI, 0.64-0.94) and a specificity of 0.88 (95\% CI, 0.69-0.97).

While the results appear to demonstrate IVC collapsibility is an acceptable surrogate for more complex measures of fluid responsiveness, multiple methodological issues weaken this conclusion. For example, a 39\% change in IVC diameter was not determined prospectively, meaning that, after obtaining the study results, the authors then identified the threshold exhibiting optimal diagnostic accuracy. Moreover, the ability of this specific threshold to yield the same diagnostic accuracy when tested in an external cohort is unknown.

A 2017 study published in Critical Care Medicine assessed the ability of IVC variation in spontaneously breathing ICU patients with sepsis to predict fluid responsiveness (2). Although the area under the ROC was similar to that demonstrated by Bortolotti et al. (0.89; 95\% CI, 0.82-0.97), the cutoff for optimal diagnostic performance was calculated at $48 \%$. Consistent with this study, a 2017 metaanalysis published in Shock found that the ideal cutoff of spontaneously breathing cohorts varied from 36-50\% (3). This heterogeneity of diagnostic accuracy for particular cIVC cutoffs among studies suggests that any single cutoff utilized in clinical practice will likely yield similarly varied results.

In addition, the results presented by Bortolotti et al. present a far more optimistic view of IVC diagnostic capabilities than the remainder of the literature. Long et al., in their review of the literature found the AUROC to be 0.76 , far less impressive than what was reported by Bortolotti et al. (3). IVC variation may perform better in patients with dysrhythmias, but the impressive diagnostic characteristics far more likely result from the small sample size and retrospectively selected threshold.

More important than quibbling over small differences in ROCs is whether fluid responsiveness itself is the most appropriate method by which to judge IVC variation performance. Although fluid responsiveness may predict increases in SV, no study has yet to demonstrated that a fluid responsiveness-guided strategy improves important patient-centered outcomes (4). Because the average duration of cardiac output increase to a fluid bolus is approximately 60-90 minutes (5), one must question whether such fleeting effects of a fluid bolus can be expected to improve important patient-centered outcomes. Strategies that encourage continued fluid administration until a patient is no longer responsive are likely to result in iatrogenic over-resuscitation $(6,7)$. Rather than focusing on fluid responsiveness, perhaps we should examine the performance of IVC variation from the perspective of fluid tolerance, or the concept that additional fluid is unlikely to harm the patient.

Regardless of the method employed, be it IVC variation or stroke volume variation, the tools to determine when to administer fluid are far less important than the processes of care that determine how we utilize them. For example, the 2006 FACCT trial, published by the ARDSNet trial group (8), conducted a 2-by-2 factorial randomized, controlled trial to compare clinical outcomes between resuscitative strategies using either CVP or a pulmonary artery catheter (PAC), as well as a conservative or liberal fluid strategy. The inability of CVP to predict fluid responsiveness is well documented in the clinical literature (9), yet the authors also found no benefit in using a PAC when compared to CVP. More importantly, they demonstrated that a fluid restrictive approach increased ventilator-free days.

In a secondary analysis published in Critical Care Medicine, Semler et al. examined the effects of liberal versus conservative treatment strategies depending on initial fluid status (10). The authors hypothesized that patients with an elevated CVP on presentation who were randomized to the conservative strategy would undergo more aggressive diuresis and thus fare significantly better than their liberal counterparts. On the contrary, they found that in patients with an initial CVP $>8 \mathrm{mmHg}$, no difference in 60-day mortality existed between the two fluid strategies. However, in patients with a CVP $<8 \mathrm{mmHg}$, the conservative strategy group demonstrated an impressive decrease in 60-day mortality $(17 \%$ vs. 36\%; $\mathrm{P}=0.005)$. When Semler et al. attempted to parse out potential causes for this unexpected benefit, they found that, although patients with a CVP $>8 \mathrm{mmHg}$ randomized to the conservative arm received a larger amount of furosemide than those in the liberal 
arm, the overall amount of fluid administered between the groups was similar. Conversely, in patients with an initial CVP $<8$, the total dose of furosemide differed little, while the total dose of IV fluids was significantly greater in the patients randomized to the liberal fluid strategy. The authors hypothesized that fluid administration, not diuretic use, influenced outcomes in critically ill mechanically ventilated patients.

Conclusions are limited by the secondary nature of this analysis, and validation is certainly warranted, but many studies published since have validated the harms of fluid administration demonstrated in the FACCT trial. For example, in the CLASSIC trial published in Intensive Care Medicine in 2016 (11), Hjortrup et al. enrolled patients $\geq 18$ years who were admitted to the ICU for sepsis with signs of severe circulatory impairment, received at least $30 \mathrm{~mL} / \mathrm{kg}$ ideal body weight (IBW) of fluid, and had pressordependent shock. Patients were randomized to either a fluid restrictive or fluid liberal strategy. Patients in the fluid restrictive group could receive a bolus of isotonic crystalloid only in cases of severe hypoperfusion, defined as either "a serum lactate of $\geq 4 \mathrm{mmol} / \mathrm{L}$, a $\mathrm{MAP}<50 \mathrm{mmHg}$ in spite of the infusion of norepinephrine, mottling beyond the edge of the kneecap, or oliguria, defined as urinary output at most $0.1 \mathrm{~mL} / \mathrm{kg}$ IBW in the last hour." Despite enhanced lactate clearance and greater urine output in early stages of their ICU stay, patients randomized to the liberal group demonstrated an increase trend in both acute kidney injury and 90-day mortality.

Another recent trial highlighting the harms of fluid administration was published in $7 A M A$ by Andrews et al. (12). The authors randomized adults presenting to Zambia's 1,500-bed national hospital with sepsis and hypotension to either standard care or a prespecified fluid resuscitation protocol. Patients randomized to the protocol arm were given an initial 2-liter bolus followed by an assessment looking for signs of fluid intolerance. Patients randomized to the protocol group received far more aggressive upfront care than their counterparts, receiving a median of 3.5 L (IQR, 2.7-4.0 L) of IV fluids compared with $2.0 \mathrm{~L}$ (IQR, 1.0-2.5 L) in the usual care group. While 38.7\% of patients in the protocol arm received $\geq 4 \mathrm{~L}$ of IV fluid within the first 6 hours of care, only $48.3 \%$ in the usual care group received any intravenous fluid bolus. Similar to the CLASSIC trial, patients randomized to receive protocolized care were rewarded with markedly faster rates of lactate clearance, but this early surrogate of optimized resuscitation failed to translate into improvements in patient-centered benefits. In fact, those in the fluid resuscitation protocol arm experienced increased in-hospital mortality $(48.1 \%$ vs. $33.0 \% ; \mathrm{P}=0.03)$.

Finally, the FEAST trial published in the NEFM in 2010 by Maitland et al. enrolled 3,141 children with septic shock in Uganda, Kenya, or Tanzania. These children were randomized to receive a bolus of normal saline, a bolus of albumin, or no bolus (13). The trial was stopped early after noting increased mortality in children randomized to either the saline or albumin arms of the trial.

Over-resuscitation rarely occurs in the spectacular fashion of our early goal-directed yesteryear. Instead, it transpires in a far more clandestine manner, in small aliquots administered surreptitiously over time. Each single bolus when examined in isolation appears harmless, but in aggregate, the resulting fluid balance can be devastating. Whether measuring jugular venous pressure in a Zambian emergency department or assessing IVC variation with bedside US in a state-of-the-art ICU, empirically pursuing a goal-directed strategy that drives patients towards the flat portion of the Frank-Starling curve without considering the underlying pathology is likely to lead to overresuscitation and downstream harms. The high-quality study by Bortolotti et al. further strengthens the evidence behind the ability of IVC variation to determine fluid responsiveness. However, the fundamental question we must address is whether fluid responsiveness should truly be our resuscitative endpoint, or are we simply decorating a sinking ship?

\section{Acknowledgements}

None.

\section{Footnote}

Conflicts of Interest: The authors have no conflicts of interest to declare.

\section{References}

1. Bortolotti P, Colling D, Colas V, et al. Respiratory changes of the inferior vena cava diameter predict fluid responsiveness in spontaneously breathing patients with cardiac arrhythmias. Ann Intensive Care 2018;8:79.

2. Preau S, Bortolotti P, Colling D, et al. Diagnostic Accuracy of the Inferior Vena Cava Collapsibility to Predict Fluid Responsiveness in Spontaneously Breathing Patients With 
Sepsis and Acute Circulatory Failure. Crit Care Med 2017;45:e290-7.

3. Long E, Oakley E, Duke T, et al. Does Respiratory Variation in Inferior Vena Cava Diameter Predict Fluid Responsiveness: A Systematic Review and Meta-Analysis. Shock 2017;47:550-9.

4. Shah MR, Hasselblad V, Stevenson LW, et al. Impact of the pulmonary artery catheter in critically ill patients: meta-analysis of randomized clinical trials. JAMA 2005;294:1664-70.

5. Nunes TS, Ladeira RT, Bafi AT, et al. Duration of hemodynamic effects of crystalloids in patients with circulatory shock after initial resuscitation. Ann Intensive Care 2014;4:25.

6. Kuan WS, Ibrahim I, Leong BS, et al. Emergency department management of sepsis patients: a randomized, goal-oriented, noninvasive sepsis trial. Ann Emerg Med 2016;67:367-78.e3.

7. Spiegel RJ, Kappler SB, McCurdy MT. Fluid responsiveness: goal-directed care or resuscitative guide to salt water drowning? Crit Care Med 2018;46:e816-7.

8. Wiedemann HP, Wheeler AP, Bernard GR, et al.

Cite this article as: Spiegel RJ, McCurdy MT. Inferior vena cava variation predicts fluid responsiveness during dysrhythmias: a rational rearrangement of chairs on The Titanic. Ann Transl Med 2018;6(Suppl 1):S67. doi: 10.21037/atm.2018.10.36
Comparison of two fluid-management strategies in acute lung injury. N Engl J Med 2006;354:2564-75.

9. Marik PE, Cavallazzi R. Does the central venous pressure predict fluid responsiveness? An updated meta-analysis and a plea for some common sense. Crit Care Med 2013;41:1774-81.

10. Semler MW, Wheeler AP, Thompson BT, et al. Impact of initial central venous pressure on outcomes of conservative versus liberal fluid management in acute respiratory distress syndrome. Crit Care Med 2016;44:782-9.

11. Hjortrup PB, Haase N, Bundgaard H, et al. Restricting volumes of resuscitation fluid in adults with septic shock after initial management: the CLASSIC randomised, parallel-group, multicentre feasibility trial. Intensive Care Med 2016;42:1695-705.

12. Andrews B, Semler MW, Muchemwa L, et al. Effect of an Early Resuscitation Protocol on In-hospital Mortality Among Adults With Sepsis and Hypotension: A Randomized Clinical Trial. JAMA 2017;318:1233-40.

13. Maitland K, Kiguli S, Opoka RO, et al. Mortality after fluid bolus in African children with severe infection. $\mathrm{N}$ Engl J Med 2011;364:2483-95. 